\title{
Erratum to: Massless Dirac equation from Fibonacci discrete-time quantum walk
}

\author{
Giuseppe Di Molfetta • Lauchlan Honter • \\ Ben B. Luo - Tatsuaki Wada - Yutaka Shikano
}

Published online: 14 May 2015

(C) Chapman University 2015

\section{Erratum to: Quantum Stud.: Math. Found. DOI 10.1007/s40509-015-0038-6}

On finalizing the publication process of the original article, we had the following mistakes and make the corrections.

1. In page 2 line 5, we had the mistake on the citation as follows: the original manuscript "of a massive Dirac fermion [39-43]" should be changed to "of a massive Dirac fermion [38-43]".

2. In page 2 line 7 , we had the mistake on the citation as follows: the original manuscript "can be regarded as quantum dynamical simulators $[39,40]$ " should be changed to "can be regarded as quantum dynamical simulators [40, 45]".

3. In page 10, we had the mistake on the double citation. References $[46,47]$ are exactly same to those of Refs. $[49,50][1,2]$, respectively. Since we did not cite Refs. $[46,47]$ in the original manuscript, we delete these.

The online version of the original article can be found under doi:10.1007/s40509-015-0038-6.

G. Di Molfetta $\cdot$ L. Honter · B. B. Luo · Y. Shikano

Research Center of Integrative Molecular Systems (CIMoS), Institute for Molecular Science,

Natural Institutes of Natural Sciences, 38 Nishigo-Naka, Myodaiji, Okazaki, Aichi 444-8585, Japan

G. Di Molfetta

LERMA, Observatoire de Paris, PSL Research University, CNRS, Sorbonne Universités,

UPMC Univ. Paris 6, UMR 8112, 75014 Paris, France

e-mail: giuseppe.dimolfetta@ens.fr

L. Honter · B. B. Luo

School of Physics, The University of Western Australia, 35 Stirling Hwy, Crawley, Perth, WA 6009, Australia

T. Wada

Department of Electrical and Electronic Engineering, Ibaraki University,

12-4-1 Nakanarusawa, Hitachi, Ibaraki 316-8511, Japan

Y. Shikano $(\bowtie)$

Institute for Quantum Studies, Chapman University, 1 University Dr., Orange, CA 92866, USA

e-mail: yshikano@ims.ac.jp 


\section{References}

1. Rohde, P.P., Brennen, G.K., Gilchrist, A.G.: Quantum walks with memory provided by recycled coins and a memory of the coin-flip history. Phys. Rev. A 87, 052302 (2013)

2. Shikano, Y., Wada, T., Horikawa, J.: Discrete-time quantum walk with feed-forward quantum coin. Sci. Rep. 4, 4427 (2014) 